\title{
Sexual hormones and mental health
}

\author{
Lucía Martínez-Mota
}

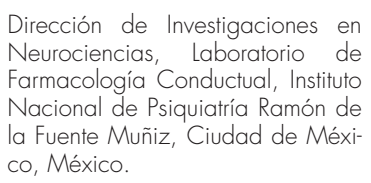

Dirección de Investigaciones en Neurociencias, Laboratorio de Farmacología Conductual, Instituto Nacional de Psiquiatría Ramón de la Fuente Muñiz, Ciudad de México, México.

Correspondence:

Lucía Martínez Mota

Dirección de Investigaciones en Neurociencias, Laboratorio de Farmacología Conductual, Instituto Nacional de Psiquiatría Ramón de la Fuente Muñiz. Calz. México-Xochimilco 101, Col. San Lorenzo Huipulco, Tlálpan, 14370, Ciudad de México, México. Phone: +52 (55) 4160 - 5054 Email: lucia@imp.edu.mx

\section{Citation:}

Martínez-Mota, L. (2019). Sexual hormones and mental health. Salud Mental, 43(1), 1-2

DOI: $10.17711 /$ SM.0185-3325.2020.001
Sexual steroids are produced by sexual glands and distributed through the blood stream towards different tissues as the brain. In turn, the brain is a steroidogenic organ (Baulieu, 1991), which increase the chances of these hormones to influence mental functions. Sexual hormones affect physiology and behavior throughout two action types. Organizational actions during developmental crucial stages lead to permanent changes, such as brain sexual differentiation. Afterwards, activational actions are established on a programmed brain leading to transient changes, such as sexual behavior (Feder, 1981). It is no coincident that many psychiatric disorders have their onset in adolescence when sexual hormones are rising and new brain networks are in formation. Sudden or slow decrease of sexual steroids in female and males, respectively, are associated with depressive symptomatology, irritability, and failures in cognition. But, on the other hand, hormones also can protect from damages to the central nervous system, as estrogens do during the women reproductive life.

Sexual hormones bind their receptors to produce long-term effects (i.e., in hours or days) mediated by genomic mechanisms, or short-term effects (i.e., in seconds) mediated by non-canonical mechanisms. Estrogen, progesterone, and androgen receptors are widely distributed in structures such as hippocampus, amygdala and prefrontal cortex (Simerly, 1993), interacting with neurotransmitter systems that regulate affection, mood, and cognition, and that participate in the response to treatments. Furthermore, gonadal hormones interrelate with those of the hypothalamus-pituitary-adrenal axis in a bidirectional manner. In a way, estrogens enhance whereas testosterone reduces corticosterone release from adrenal glands, buffering stress effects; but, in the other way, corticosterone levels during prolonged stress could reduce testosterone concentration, thus affecting reproductive, social, emotional, and cognitive functions mediated by androgens (Handa, Burgess, Kerr, \& O'Keefe, 1994).

Current research focuses on understanding complex interrelations between sexual hormones, neurotransmitter systems, and circuits that participate in expression of behavior in normal or pathological conditions to translate this knowledge into practical use for treatment or prevention of psychiatric disorders. In this sense, hormonal alterations are common in patients with psychiatric disorders before they receive treatment (Margolese, 2000), and these and other disturbances (e.g., metabolic ones) are elicited by drugs prescribed for relieving psychiatric symptoms (Reynolds \& Kirk, 2010). Notwithstanding, current psychiatric practice do not considers sex hormones disturbances as possible mediators of symptomatology or treatment response (personal communication, Flores-Ramos). In this regard, evaluation of endocrinological aspects in psychiatry has never been more important as in recent years.

The present compilation of papers is designed to show recent advances in the field by presenting original articles from a preclinical and clinical research. A summary of the most important findings is presented below, and grouped according to the theme.

Juan Francisco Rodríguez-Landa et al. present the study "Actions of progesterone on depression-like behavior in a model of surgical menopause are mediated by GABA receptors." Results show that females rats with a long-term hormone deprivation period are still sensitive to hormonal restitution with progesterone, eliciting antidepressant-like effects. Progesterone and its $5 \alpha$-reduced metabolite, allopregnanolone, are neurosteroids participating in recovery of homeostasis after stress exposure. Such role of progesterone 
agrees with the present results. Furthermore, the blockade of progesterone effects with picrotoxin strengthen the relationship of GABAergic system with female depression, an issue studied by different research teams in the world.

Evidence has shown that hormones modify the effect of psychiatric treatments. Adriana Álvarez-Silva and Alonso Fernández-Guasti, in their paper "Does the antidepressant-like effect of mirtazapine and venlafaxine differ between male and female rats?," describe that male rodents exhibited antidepressant effects associated with an increase in serotonergic and noradrenergic activity. Hormone-primed females only showed behaviors associated with an increased serotonergic neurotransmission, suggesting that estrogens favor serotonergic actions of both antidepressants. Susana Medina-Loera et al. in their paper "Association between prolactin serum levels and cognitive function in chronic schizophrenia patients," showed that men were more vulnerable to some side-effects of antipsychotics. They studied a sample of 31 patients coursing with 11.5 years of disorder evolution and monotherapy in most participants. Only in male patients a negative moderated correlation was observed between cognition functions and serum prolactin levels. Altogether, these papers suggest sex differences in some effects of antidepressant and antipsychotics, and hypothesize that female hormones would act as protection factors, i.e., buffering side-effects of some drugs.

A decrease in androgen levels has been associated with low sexual motivation, but brain substrates underlying such changes have been scarcely explored. Marisela Hernández González et al., in their paper "Electroencephalographic changes and testosterone levels in a pubertal stress animal model: effects on adult sexual motivation," show that social isolation from puberty changed the functionality of neuronal circuits that participates in the expression of sexual motivated behaviors. Chronically stressed males had increased activity of basolateral amygdala in the presence of sexually receptive females. Disturbances in stressed males were associated to hypogonadism suggesting that normal androgen levels modulate amygdala activity, thus favoring cognitive processing of sexual stimuli by medial prefrontal cortex, as it was observed in non-stressed males.

Hormones and metabolic factors interact in complex manners modifying behavior. In the paper "Evaluation of hormonal and metabolic factors related to depression in reproductive age women," Mónica Flores-Ramos et al. studied 40 females, 23 with MDD diagnosis and 17 controls, with age ranging from 19 to 46 years old, and with regular menstrual cycles. Authors found that more intense depression symptoms were related to lower testosterone levels and higher serum glucose levels. Interestingly, the paper "Diabetic female rats exhibit defensive aggression during mating," by Abigail Hernández-Munive et al., describes that hyperglycemia increases defensive, but not offensive, aggressive behavior, interfering with copulation. Neuropathic pain and, to greater extent, increased anxiety and lower brain serotonin levels, may explain the present findings. Insulin treatment reduced expression of aggressive behavior and may have restored females' ability for social and sexual interaction. Summarizing, these studies highlight the importance of evaluation of hormones and glycemic control in women suffering psychiatric disorders, with implications for their treatment.

I hope these studies encourage physicians, psychiatrists, and specialist in mental health to take into account the multiple factors involved in mental health, including sexual hormones. I also hope current results stimulate researchers to raise new questions that lead to new and better interventions to improve mental health.

\section{REFERENCES}

Baulieu, É. É. (1991). Neurosteroids: a new function in the brain. Biology of the Cell, 7l(1-2), 3-10. doi: 10.1016/0248-4900(91)90045-O

Feder, H. H. (1981). Perinatal hormones and their role in the development of sexually dimorphic behaviors. In Adler, N. T. (Ed.) Neuroendocrinology of Reproduction. Physiology and Behavior. New York: Plenum Press.

Handa, R. J., Burgess, L. H., Kerr, J. E., \& O'Keefe, J. A. (1994). Gonadal steroid hormone receptors and sex differences in the hypothalamo-pituitary-adrenal axis. Hormones and Behavior, 28(4), 464-476. doi: 10.1006/hbeh.1994.1044

Margolese, H. C. (2000). The male menopause and mood: testosterone decline and depression in the aging male - Is there a link?. Journal of Geriatric Psychiatry and Neurology, 13(2), 93-101. doi: 10.1177/089198870001300208

Reynolds, G. P., \& Kirk, S. L. (2010). Metabolic side effects of antipsychotic drug treatment - pharmacological mechanisms. Pharmacology \& Therapeutic, 125(1), 169-179. doi: 10.1016/j.pharmthera.2009.10.010

Simerly, R. B. (1993). Distribution and regulation of steroid hormone receptor gene expression in the central nervous system. Advances in Neurology, 59, 207-226. 